\title{
Childhood L1 Acute Lymphoblastic Leukemia
}

National Cancer Institute

\section{Source}

National Cancer Institute. Childhood L1 Acute Lymphoblastic Leukemia. NCI Thesaurus.

Code C114560.

L1 acute lymphoblastic leukemia that occurs during childhood. 